\title{
Second-order cone AC optimal power flow: convex relaxations and feasible solutions
}

\author{
${\text { Zhao } \text { YUAN }^{1}{ }^{\circledR} \text {, Mohammad Reza HESAMZADEH }}^{1}$
}

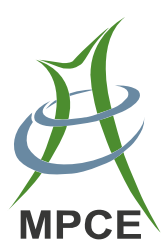

\begin{abstract}
Optimal power flow (OPF) is the fundamental mathematical model to optimize power system operations. Based on conic relaxation, Taylor series expansion and McCormick envelope, we propose three convex OPF models to improve the performance of the second-order cone alternating current OPF (SOC-ACOPF) model. The underlying idea of the proposed SOC-ACOPF models is to drop assumptions of the original SOC-ACOPF model by convex relaxation and approximation methods. A heuristic algorithm to recover feasible ACOPF solution from the relaxed solution of the proposed SOC-ACOPF models is developed. The proposed SOC-ACOPF models are examined through IEEE case studies under various load scenarios and power network congestions. The quality of solutions from the proposed SOC-ACOPF models is evaluated using MATPOWER (local optimality) and LINDOGLOBAL (global optimality). We also compare numerically the proposed SOC-ACOPF models with other two convex ACOPF models in the literature. The numerical results show robust performance of the proposed SOCACOPF models and the feasible solution recovery algorithm.
\end{abstract}

CrossCheck date: 19 July 2018

Received: 19 March 2018/ Accepted: 19 July 2018 / Published online: 15 October 2018

(C) The Author(s) 2018

$\triangle$ Zhao YUAN

yuanzhao@kth.se

Mohammad Reza HESAMZADEH

mrhesamzadeh@kth.se

1 Department of Electric Power and Energy Systems, KTH Royal Institute of Technology, Teknikringen 33, 11428 Stockholm, Sweden
Keywords Optimal power flow, Conic relaxation, McCormick envelope, Taylor series expansion, Feasible solution

\section{Introduction}

Optimal power flow (OPF) is an indispensable tool in fairly wide areas of power system operations and the applications are still expanding [1-4]. The challenges of integrating large amount of renewable energy, increasing multi-terminal high voltage direct current (HVDC) connections and growing number of prosumers in distribution grid are now pushing the electricity industry to seek more accurate, reliable and efficient OPF tools. Since the alternating current (AC) power flow constraints are complex, nonlinear and nonconvex in nature, enormous research efforts have been put into developing efficient algorithms to solve OPF during the past decades. References [1] and [2] summarize methods to solve OPF in the early stages ranging from linear, nonlinear and quadratic programming to Newton-based algorithm and interior point method (IPM). Heuristic optimization algorithms based on evolutionary and intelligence approaches to solve OPF can be found in [5] and [6]. Traditionally, the direct current OPF (DCOPF) as an estimation of full alternating current OPF (ACOPF) is pervasively employed for large-scale power system calculations [7]. With fast development of smart grids [8], distribution network is now in the unprecedented interest of advanced monitoring and control [9]. Distribution networks have larger resistance to reactance $(R / X)$ ratio as compared to the transmission networks. Accordingly, the DCOPF results of distribution networks need to be carefully examined. Besides, the operation points and nodal prices obtained from solving ACOPF in transmission 
and distribution networks are more accurate $[10,11]$. Accordingly, accurate and fast methods to solve ACOPF are in demand.

Mathematical modelling techniques to convexify the nonconvex ACOPF model by convex relaxations can give useful bound of ACOPF objective function. Convexity also guarantees finding global solution by using mature solution algorithms (e.g. IPM). Considering abundant commercially available solvers (such as MOSEK [12]) for solving convex optimization problems, the remaining task is to tighten the relaxations used in the convexification. Though assuming no lower bound for active power generation to exactly convexify mesh networks is not realistic, the proposed branch flow model in [13] as a convex relaxation of ACOPF is very important and useful. Reference [14] gives exact convex relaxations of OPF under some assumptions on network parameters. Based on the branch flow approach, authors in [15] present an ACOPF model using second-order cone programming (SOCP) and it shows accurate solutions for several IEEE test cases when the objective is transmission loss minimization. The SOCP based convex ACOPF model in [15] serves as the starting point of deriving our second-order cone ACOPF (SOCACOPF) models in this paper. A cone-programming-based OPF for radial distribution networks is proposed in [16]. Reference [9] continues to improve its cone relaxation by generating tight cutting planes. For radial networks, sufficient conditions regarding network property and voltage upper bound under which the proposed relaxed ACOPF can give global ACOPF solution are derived in [14]. Recent applications of SOCP based convex ACOPF model in distributional locational marginal pricing (DLMP), transmission-distribution coordination and decentralized power system operation can be found in [17-19].

Semi-definite programming (SDP) is another promising convexification approach for ACOPF [20, 21]. The computational limits of SDP are shown in [22]. Efficient algorithms for solving SDP-based ACOPF model remain to be found [20]. Regarding solving ACOPF in large-scale power networks, SDP-based ACOPF takes much more CPU time than SOCP-based ACOPF. Using matrix combination and decomposition techniques, authors in [23] accelerate SDP-based ACOPF and show that more than 1000 seconds solver time are required to compute cases with around 3000 buses. Important analysis and results from [24-26] show that SDP relaxations are exact only for limited types of problems. Even for a 2-bus 1-generator power system, SDP-based ACOPF can be infeasible and inexact [27]. In cases where the exactness is not guaranteed, solutions of SDP-based ACOPF rarely have physical meanings.

Results from [28] show that quadratic convex (QC) relaxation of ACOPF may produce some improvements in accuracy over SOCP-based ACOPF but with reduced computational efficiency. The feasible region relationship of SOCP, SDP and QC approaches are analyzed in [28]. The SDP and QC approaches give tighter relaxations than the SOCP approach but they are not equivalent to each other. In terms of computational performance, the QC and SOCP approaches are much faster and reliable than the SDP approach [28]. Based on first-order Taylor series expansion, a current voltage (IV) formulation for ACOPF is proposed in [29, 30]. The advantages of the currentvoltage formulation are that the approximated ACOPF problem is linear and much faster to be solved than nonlinear formulations. However, an iterative algorithm is required to check the violations of nonconvex ACOPF constraints and then to construct the inner or outer bounds of the approximations. Authors in [31] propose three different relaxation methods to improve SOCP-based ACOPF. The arctangent constraints in the rectangular formulation of ACOPF are convexified by McCormick relaxations, polyhedral envelopes and dynamically generated linear inequalities [31]. The results show prominent computational efficiency of the SOCP approach over the SDP approach [31]. Compared with the formulations in [31], the proposed SOC-ACOPF models in this paper deal with the nonconvex ACOPF constraints directly and the set of decision variables used in our models are different.

Regarding the feasibility of the relaxed solutions, three types of sufficient conditions about power injections, voltage magnitudes and phase angles to guarantee obtaining exact solutions are proposed in [26]. Authors in [31] strengthen the relaxations of SOCP-based ACOPF model by dynamically generating linear valid inequalities to separate solutions of SOCP-based ACOPF from other relaxed constraints. However, it is not guaranteed that feasible solution can be always recovered by this approach. The complementarity conditions in the Karush-KuhnTucker (KKT) system of DCOPF are used in [32] to recover feasible solution of ACOPF. A sequential algorithm to improve the tightness of some relaxed constraints of a SOCP-based ACOPF model is proposed in [17]. The performance of this algorithm for large power networks remains to be improved.

The main contributions of the current paper are threefold:

1) Three SOC-ACOPF models based on second-order cone relaxations, Taylor series expansion and McCormick envelops are proposed.

2) A heuristic algorithm is proposed to recover feasible solutions of ACOPF from the relaxed solutions obtained from the proposed SOC-ACOPF models.

3) A computational comparison with other SOCP-based ACOPF formulations in the literature is conducted. 
Note we are claiming that the derived SOC-ACOPF models are the original contributions of this paper (not the SOCP method).

The rest of this paper is organized as follows. Section 2 presents mathematical formulations of the proposed SOCACOPF models. Section 3 proposes the heuristic algorithm to recover feasible solutions. Section 4 discusses numerical results for various IEEE test cases under different load scenarios and power network congestions. A numerical comparison with two other SOCP based convex ACOPF models in the literature is conducted. Section 5 concludes the improvements of the proposed SOC-ACOPF models.

\section{SOC-ACOPF}

It is assumed that the electric networks are three phase and balanced (this assumption is generally used by many OPF models such as the DCOPF model). Our proposed SOC-ACOPF models use less approximations or assumptions than the DCOPF model. Considering the wide application of DCOPF, the proposed SOC-ACOPF models are more applicable. The proposed SOC-ACOPF models are derived using line sending-end power injections and voltage phase angle difference variables. In this way, we can directly obtain voltage phase angle solutions from the models. Note that in some of the derived models, instead of using voltage magnitude variables $v_{n}, v_{s_{l}}, v_{r_{l}}$, voltage magnitude square variables $V_{n} \triangleq v_{n}^{2}, V_{s_{l}} \triangleq v_{s_{l}}^{2}, V_{r_{l}} \triangleq v_{r_{l}}^{2}$ are included (voltage magnitude can be recovered from the model by taking the root of the voltage magnitude square solutions). $n \in N$ is the index of node set $N, l$ is the index of transmission line set $L . v_{n}$ is the voltage magnitude at node n. $v_{s_{l}}, v_{r_{l}}$ are the voltage magnitudes at the sending end and receiving end of transmission line $l$. The convexity of the proposed SOC-ACOPF models are further validated numerically by MOSEK solver which can only solve convex programming models in general algebraic modeling system (GAMS). We want to emphasize that the numerical validation by MOSEK is a double-check of the convexity of the proposed SOC-ACOPF models. We have detailed analytic explanations in following sections about why our derived model are convex. In short, the fundamental reason is because all the derived constraints are either linear or in the form of second-order cone which are definitely convex constraints.

\subsection{SOC-ACOPF: Model P}

Model P of the SOC-ACOPF is set out in (1)-(10) [15]. Note $p_{s_{l}}, q_{s_{l}}$ represent receiving end power flows in [15] which are different in our formulation (sending end active and reactive power flows). So some constraints are accordingly different. The term $s$ in $p_{s_{l}}, q_{s_{l}}, v_{s_{l}}, V_{s_{l}}$ is not an index but only to imply the meaning of sending end of line $l$. The term $r$ in $v_{r_{l}}, V_{r_{l}}$ is not an index but only to imply the meaning of receiving end of line $l$. The term $d$ in $p_{d_{n}}, q_{d_{n}}$ is not an index but only to imply the meaning of power demand. $p_{d_{n}}, q_{d_{n}}$ are active and reactive power demand (parameters). Similar reasoning holds for the term $o$ in $p_{o_{l}}, q_{o_{l}}$ which is to denote the meaning of power loss. $p_{o_{l}}, q_{o_{l}}$ are active and reactive power loss.

$\min _{\Omega} f\left(p_{n}, q_{n}, p_{o_{l}}, q_{o_{l}}\right)$

s.t.

$p_{n}-P_{d_{n}}=\sum_{l \in L}\left(A_{n l}^{+} p_{s_{l}}-A_{n l}^{-} p_{o_{l}}\right)+G_{n} V_{n} \quad \forall n \in N$

$q_{n}-Q_{d_{n}}=\sum_{l \in L}\left(A_{n l}^{+} q_{s_{l}}-A_{n l}^{-} q_{o_{l}}\right)-B_{n} V_{n} \quad \forall n \in N$

$K_{l} \geq p_{o_{l}} \geq \frac{p_{s_{l}}^{2}+q_{s_{l}}^{2}}{V_{s_{l}}} R_{l} \quad \forall l \in L$

$p_{o_{l}} X_{l}=q_{o_{l}} R_{l} \quad \forall l \in L$

$V_{s_{l}}-V_{r_{l}}=2 R_{l} p_{s_{l}}+2 X_{l} q_{s_{l}}-R_{l} p_{o_{l}}-X_{l} q_{o_{l}} \quad \forall l \in L$

$\theta_{l}=X_{l} p_{s_{l}}-R_{l} q_{s_{l}} \quad \forall l \in L$

$V_{\min , n} \leq V_{n} \leq V_{\max , n} \quad \forall n \in N$

$p_{\min , n} \leq p_{n} \leq p_{\max , n} \quad \forall n \in N$

$q_{\min , n} \leq q_{n} \leq q_{\max , n} \quad \forall n \in N$

where $\Omega=\left\{p_{n}, q_{n}, p_{s_{l}}, q_{s_{l}}, p_{o_{l}}, q_{o_{l}}, V_{n}, \theta_{l}\right\} \in \mathbf{R}$ is the set of decision variables. Equations (2) and (3) represent the active and reactive power balance. $A_{n l}^{+}$and $A_{n l}^{-}$are elements of incidence matrix of the network with $A_{n l}^{+}=1, A_{n l}^{-}=0$ if $n$ is the sending end of line $l$ and $A_{n l}^{+}=-1, A_{n l}^{-}=-1$ if $n$ is the receiving end of line $l$. Since we allow the variables $p_{s_{l}}, q_{s_{l}}$ to take both positive and negative values, the default power flow direction of each line does not affect the final results (negative values of these variables mean the actual power flow direction is in the reverse direction of the default power flow direction). $R_{l}$ is the resistant of line $l . X_{l}$ is the reactance of line $l$. $V_{\min , n}, V_{\max , n}$ are the lower and upper bounds of $V_{n}$. $p_{\min , n}, p_{\max , n}$ are the lower and upper bounds of $p_{n}$. $q_{\min , n}, q_{\max , n}$ are lower and upper bounds of $q_{n} . K_{l}$ is the upper bound of $p_{o_{l}}$. Model $\mathrm{P}$ is convex because: (1) constraints (2), (3) and (5)-(10) are linear; (2) constraint (4) is in the form of second-order cone. Constraints (4) and (5) represent active power and reactive power loss. The left side of (4) bounds $p_{o_{l}}$ (which equivalently bounds capacity of line $l$ ). $\theta_{l} \triangleq \theta_{s_{l}}-\theta_{r_{l}}$ is defined as the voltage phase angle difference of line $l . \theta_{s_{l}}, \theta_{r_{l}}$ 
are the voltage phase angles at the sending end and receiving end of transmission line $l$. Equation (7) is approximated from the nonconvex constraint (11) as below:

$v_{s_{l}} v_{r_{l}} \sin \theta_{l}=X_{l} p_{s_{l}}-R_{l} q_{s_{l}} \quad \forall l \in L$

This approximation is based on following assumptions: (1) assumption 1, voltage magnitude product $v_{s_{l}} v_{r_{l}}$ is approximately equal to 1 per-unit in (11); (2) assumption 2, voltage phase angle difference across each line is small enough such that $\sin \theta_{l} \approx \theta_{l}$.

It is worth to mention that assumption 1 is only used to linearize constraint (11). Voltage solutions from Model P are allowed to take other values which do not satisfy assumption 1. In this paper, we refer Model $\mathrm{P}$ summarized in (1)-(10) as the original SOC-ACOPF model. We will gradually improve Model $\mathrm{P}$ by dropping assumptions 1 and 2 in following sections. Constraint (7) in Model P is replaced by different formulations in the new SOC-ACOPF models. Assumptions 1 and 2 are valid for both transmission and distribution network in normal operations. However, there can be abnormal situations when these assumptions do not hold. This is one of the reasons that we want to drop assumptions 1 and 2, and propose new SOCACOPF models in order to improve the solution accuracy.

\subsection{SOC-ACOPF: Model R}

To drop assumption 1 in Model P, we use the following bilinear transformation:

$v_{s_{l}} v_{r_{l}}=\frac{1}{4}\left[\left(v_{s_{l}}+v_{r_{l}}\right)^{2}-\left(v_{s_{l}}-v_{r_{l}}\right)^{2}\right] \quad \forall l \in L$

If we introduce auxiliary variable $v_{m_{l}}$ as (note the term $m$ in the subscript of $v_{m l}$ is not an index):

$v_{m_{l}}=v_{s_{l}} v_{r_{l}} \quad \forall l \in L$

And repeat transformation (12) then the left side of (11) can be replaced by:

$v_{m_{l}} \theta_{l}=\frac{1}{4}\left[\left(v_{m_{l}}+\theta_{l}\right)^{2}-\left(v_{m_{l}}-\theta_{l}\right)^{2}\right] \quad \forall l \in L$

Introducing new variables $u_{x_{l}}, w_{x_{l}}, u_{v_{l}}$ and $w_{v_{l}}$ as (note the terms $x$ and $v$ in the subscripts are not indexes but are only to distinguish different variables):

$$
\begin{array}{ll}
u_{x_{l}}=v_{s_{l}}+v_{r_{l}} & \forall l \in L \\
w_{x_{l}}=v_{s_{l}}-v_{r_{l}} & \forall l \in L \\
u_{v_{l}}=v_{m_{l}}+\theta_{l} & \forall l \in L \\
w_{v_{l}}=v_{m_{l}}-\theta_{l} & \forall l \in L
\end{array}
$$

Equations (12) and (14) can be expressed by the new variables $u_{x_{l}}, w_{x_{l}}, u_{v_{l}}$ and $w_{v_{l}}$ as:

$$
\begin{array}{ll}
v_{s_{l}} v_{r_{l}}=\frac{1}{4}\left(u_{x_{l}}^{2}-w_{x_{l}}^{2}\right) & \forall l \in L \\
v_{m_{l}} \theta_{l}=\frac{1}{4}\left(u_{v_{l}}^{2}-w_{v_{l}}^{2}\right) & \forall l \in L
\end{array}
$$

The quadratic functions $u_{x_{l}}^{2}, w_{x_{l}}^{2}, u_{v_{l}}^{2}$ and $w_{v_{l}}^{2}$ are relaxed as following second-order cones:

$$
\begin{array}{ll}
u_{x a_{l}} \geq u_{x_{l}}^{2} & \forall l \in L \\
w_{x a_{l}} \geq w_{x_{l}}^{2} & \forall l \in L \\
u_{v a_{l}} \geq u_{v_{l}}^{2} & \forall l \in L \\
w_{v a_{l}} \geq w_{v_{l}}^{2} & \forall l \in L
\end{array}
$$

where $u_{x a_{l}}, w_{x a_{l}}, u_{v a_{l}}, w_{v a_{l}}$ are auxiliary approximation variables. Note the terms $x a$ and $v a$ in the subscripts of the corresponding variables are not indexes but are only to distinguish different approximation variables.

The upper bounds are expressed linearly:

$$
\begin{array}{lc}
u_{x a_{l}} \leq\left(\bar{u}_{x_{l}}+\underline{u}_{x_{l}}\right) u_{x_{l}}-\bar{u}_{x_{l}} \underline{u}_{x_{l}} \quad \forall l \in L \\
w_{x a_{l}} \leq\left(\bar{w}_{x_{l}}+\underline{w}_{x_{l}}\right) w_{x_{l}}-\bar{w}_{x_{l}} \underline{w}_{x_{l}} \quad \forall l \in L \\
u_{v a_{l}} \leq\left(\bar{u}_{v_{l}}+\underline{u}_{v_{l}}\right) u_{v_{l}}-\bar{u}_{v_{l}} \underline{u}_{v_{l}} \quad \forall l \in L \\
w_{v a_{l}} \leq\left(\bar{w}_{v_{l}}+\underline{w}_{v_{l}}\right) w_{v_{l}}-\bar{w}_{v_{l}} \underline{w}_{v_{l}} \quad \forall l \in L
\end{array}
$$

where constraints (21)-(24) are second-order cones and constraints (25)-(28) are McCormick envelopes. $\bar{u}_{x_{l}}, \bar{w}_{x_{l}}, \bar{u}_{v_{l}}$ and $\bar{w}_{v_{l}}$ are upper bounds of the corresponding variables. $\underline{u}_{x_{l}}, \underline{w}_{x_{l}}, \underline{u}_{v_{l}}$ and $\underline{w}_{v_{l}}$ are lower bounds of the corresponding variables. The variables $v_{s_{l}}$ and $v_{r_{l}}$ are linked to their squares $V_{s_{l}}$ and $V_{r_{l}}$ by following convex constraints:

$$
\begin{aligned}
& V_{n} \geq v_{n}^{2} \quad \forall n \in N \\
& V_{n} \leq\left(\bar{v}_{n}+\underline{v}_{n}\right) v_{n}-\bar{v}_{n} \underline{v}_{n} \quad \forall n \in N
\end{aligned}
$$

where $\bar{v}_{n}$ and $\underline{v}_{n}$ are upper and lower bounds of voltage magnitude. Constraint (30) tightens the cone relaxations in (29). In Model R of SOC-ACOPF, we replace constraint (7) of optimization problem (1)-(10) by constraints (15)(18), (21)-(30) and constraints (19), (20) where the quadratic functions $u_{x_{l}}^{2}, w_{x_{l}}^{2}, u_{v_{l}}^{2}$ and $w_{v_{l}}^{2}$ are replaced by $u_{x a_{l}}, w_{x a_{l}}, u_{v a_{l}}$ and $w_{v a_{l}}$, the term $v_{s_{l}} v_{r_{l}}$ is replaced by $v_{m l}$, the term $v_{m_{l}} \theta_{l}$ is replaced by $X_{l} p_{s_{l}}-R_{l} q_{s_{l}}$. Model $\mathrm{R}$ is summarized as following:

$$
\min _{\Omega} f\left(p_{n}, q_{n}, p_{o_{l}}, q_{o_{l}}\right)
$$

s.t.

(2)-(6), (8)-(10), (15)-(18), (21)-(30)

$v_{m_{l}}=\frac{1}{4}\left(u_{x a_{l}}-w_{x a_{l}}\right) \quad \forall l \in L$ 
$X_{l} p_{s_{l}}-R_{l} q_{s_{l}}=\frac{1}{4}\left(u_{a v_{l}}-w_{a v_{l}}\right) \quad \forall l \in L$

Model R is convex because: (1) constraints (15)-(18), (21)-(28), (30)-(33) are linear; (2) constraint (29) is in the form of second-order cone. For the convexity of other constraints, please refer to the explanations of Model $\mathrm{P}$ in Section 2.1.

\subsection{SOC-ACOPF: Model T}

To drop assumption 2 of Model R, we propose to apply Taylor series expansion to approximate sine function:

$\sin \theta_{l}=\theta_{l}-\frac{\theta_{l}^{3}}{6}+\frac{\theta_{l}^{5}}{120}+O\left(\theta_{l}^{7}\right) \quad \forall l \in L$

As a trade-off between model complexity and accuracy, fifth-order Taylor series expansion is selected. The approximation error is less than $0.45 \%$ for $\left|\theta_{l}\right|<\pi / 2$. Repeating the bilinear transformation procedure similar in Section 2.2 of this paper, we have:

$u_{\theta_{l}^{2}}=\theta_{l}^{2} \quad \forall l \in L$

$u_{\theta_{l}^{3}}=\theta_{l}^{3} \quad \forall l \in L$

$u_{\theta_{l}^{5}}=\theta_{l}^{5} \quad \forall l \in L$

$\theta_{l}^{3}=\frac{1}{4}\left[\left(\theta_{l}+u_{\theta_{l}^{2}}\right)^{2}-\left(\theta_{l}-u_{\theta_{l}^{2}}\right)^{2}\right] \quad \forall l \in L$

$\theta_{l}^{5}=\frac{1}{4}\left[\left(u_{\theta_{l}^{2}}+u_{\theta_{l}^{3}}\right)^{2}-\left(u_{\theta_{l}^{2}}-u_{\theta_{l}^{3}}\right)^{2}\right] \quad \forall l \in L$

We introduce auxiliary variables to formulate relaxations of quadratic equations:

$$
\begin{array}{ll}
h_{x_{l}}=\theta_{l}+u_{\theta_{l}^{2}} \quad \forall l \in L & \\
y_{x_{l}}=\theta_{l}-u_{\theta_{l}^{2}} \quad \forall l \in L & \\
h_{v_{l}}=u_{\theta_{l}^{2}}+u_{\theta_{l}^{3}} \quad \forall l \in L & \\
y_{v_{l}}=u_{\theta_{l}^{2}}-u_{\theta_{l}^{3}} \quad \forall l \in L & \forall l \in L \\
u_{v_{l}}=v_{m_{l}}+\left(\theta_{l}-\frac{u_{\theta_{l}^{3}}}{6}+\frac{u_{\theta_{l}^{5}}}{120}\right) & \forall l \in L \\
w_{v_{l}}=v_{m_{l}}-\left(\theta_{l}-\frac{u_{\theta_{l}^{3}}}{6}+\frac{u_{\theta_{l}^{5}}}{120}\right) &
\end{array}
$$

Higher order terms of $\theta_{l}$ can be expressed similarly by introducing new auxiliary variables. Again, as in Model R, using variables $u_{v_{l}}$ and $w_{v_{l}}$, we have:

$u_{\theta_{l}^{3}}=\frac{1}{4}\left(h_{x_{l}}^{2}-y_{x_{l}}^{2}\right) \quad \forall l \in L$ $u_{\theta_{l}^{5}}=\frac{1}{4}\left(h_{v_{l}}^{2}-y_{v_{l}}^{2}\right) \quad \forall l \in L$

$v_{s_{l}} v_{r_{l}} \sin \theta_{l} \approx \frac{1}{4}\left(u_{v_{l}}^{2}-w_{v_{l}}^{2}\right) \quad \forall l \in L$

Similarly, auxiliary variables $h_{x a_{l}}, y_{x a_{l}}, h_{v a_{l}}$ and $y_{v a_{l}}$ are proposed:

$\begin{array}{ll}h_{x a_{l}} \geq h_{x_{l}}^{2} & \forall l \in L \\ y_{x a_{l}} \geq y_{x_{l}}^{2} & \forall l \in L \\ h_{v a_{l}} \geq h_{v_{l}}^{2} & \forall l \in L \\ y_{v a_{l}} \geq y_{v_{l}}^{2} & \forall l \in L\end{array}$

The quadratic functions are upper bounded by:

$h_{x a_{l}} \leq\left(\bar{h}_{x_{l}}+\underline{h}_{x_{l}}\right) h_{x_{l}}-\bar{h}_{x_{l}} \underline{h}_{x_{l}} \quad \forall l \in L$

$y_{x a_{l}} \leq\left(\bar{y}_{x_{l}}+\underline{y}_{x_{l}}\right) y_{x_{l}}-\bar{y}_{x_{l}} \underline{y}_{x_{l}} \quad \forall l \in L$

$h_{v a_{l}} \leq\left(\bar{h}_{v_{l}}+\underline{h}_{v_{l}}\right) h_{v_{l}}-\bar{h}_{v_{l}} \underline{h}_{v_{l}} \quad \forall l \in L$

$y_{v a_{l}} \leq\left(\bar{y}_{v_{l}}+\underline{y}_{v_{l}}\right) y_{v_{l}}-\bar{y}_{v_{l}} \underline{y}_{v_{l}} \quad \forall l \in L$

where $\bar{h}_{x_{l}}, \bar{y}_{x_{l}}, \bar{h}_{v_{l}}$ and $\bar{y}_{v_{l}}$ are upper bounds of the corresponding variables; parameters $\underline{h}_{x_{l}}, \underline{y}_{x_{l}}, \underline{h}_{v_{l}}$ and $\underline{y}_{v_{l}}$ are lower bounds of the corresponding variables. $u_{\theta_{l}^{2}}$ is bounded as follows:

$u_{\theta_{l}^{2}} \geq \theta_{l}^{2} \quad \forall l \in L$

$u_{\theta_{l}^{2}} \leq \bar{\theta}_{l}^{2} \quad \forall l \in L$

where $\bar{\theta}_{l}^{2}$ is the upper bound of $\theta_{l}^{2}$. In Model T of SOCACOPF, we replace constraint (7) of optimization problem (1)-(10) by constraints (15), (16), (21)-(28), (40)-(45), (49)-(58) and constraints (19), (46)-(48) where quadratic functions $u_{x_{l}}^{2}, w_{x_{l}}^{2}, h_{x_{l}}^{2}, y_{x_{l}}^{2}, h_{v_{l}}^{2}, y_{v_{l}}^{2}, u_{v_{l}}^{2}$ and $w_{v_{l}}^{2}$ are replaced by $u_{x a_{l}}, w_{x a_{l}}, h_{x a_{l}}, y_{x a_{l}}, h_{v a_{l}}, y_{v a_{l}}, u_{v a_{l}}$ and $w_{v a_{l}}$, the term $v_{s_{l}} v_{r_{l}}$ is replaced by $v_{m_{l}}$, the term $v_{s_{l}} v_{r_{l}} \sin \theta_{l}$ is replaced by $X_{l} p_{s_{l}}-R_{l} q_{s_{l}}$. Model $\mathrm{T}$ is summarized as following:

$\min _{\Omega} f\left(p_{n}, q_{n}, p_{o_{l}}, q_{o_{l}}\right)$

s.t.

(2)-(6), (8)-(10), (15)-(16), (21)-(28), (32), (33), (40)(45), (49)-(58)

$u_{\theta_{l}^{3}}=\frac{1}{4}\left(h_{x a_{l}}-y_{x a_{l}}\right) \quad \forall l \in L$

$u_{\theta_{l}^{5}}=\frac{1}{4}\left(h_{v a_{l}}-y_{v a_{l}}\right) \quad \forall l \in L$

Model $\mathrm{T}$ is convex because: (1) constraints (40)-(45), (53)-(56), (58)-(61) are linear; (2) constraints (49)-(52) and (57) are in the form of second-order cone. For the convexity 
of other constraints, please refer to the explanations of Model $\mathrm{P}$ in Section 2.1 and Model R in Section 2.2.

\subsection{SOC-ACOPF: Model E}

The Nonconvex term in the left side of (11) can be directly replaced by McCormick envelopes described in [33]. Employing (13) and introducing new variables $z_{\theta_{l}}=$ $\sin \theta_{l}$ and $z_{h_{l}}=v_{s} v_{r} \sin \theta_{l}$ (note the term $\theta$ in the subscript of $z_{\theta_{l}}$ and the term $h$ in the subscript of $z_{h_{l}}$ are not indexes but are only to distinguish different variables), we have:

$v_{s_{l}} v_{r_{l}} \sin \theta_{l}=v_{m_{l}} z_{\theta_{l}} \quad \forall l \in L$

$z_{h_{l}}=X_{l} p_{s_{l}}-R_{l} q_{s_{l}} \quad \forall l \in L$

McCormick envelopes for $z_{h_{l}}=v_{m_{l}} z_{\theta_{l}}$ are:

$z_{h_{l}} \geqslant \underline{v}_{m_{l}} z_{\theta_{l}}+\underline{z}_{\theta_{l}} v_{m_{l}}-\underline{v}_{m_{l}} \underline{z}_{\theta_{l}} \quad \forall l \in L$

$z_{h_{l}} \geqslant \bar{v}_{m_{l}} z_{\theta_{l}}+\bar{z}_{\theta_{l}} v_{m_{l}}-\bar{v}_{m_{l}} \bar{z}_{\theta_{l}} \quad \forall l \in L$

$z_{h_{l}} \leqslant \underline{v}_{m_{l}} z_{\theta_{l}}+\bar{z}_{\theta_{l}} v_{m_{l}}-\underline{v}_{m_{l}} \bar{z}_{\theta_{l}} \quad \forall l \in L$

$z_{h_{l}} \leqslant \bar{v}_{m_{l}} z_{\theta_{l}}+\underline{z}_{\theta_{l}} v_{m_{l}}-\bar{v}_{m_{l}} \underline{z}_{\theta_{l}} \quad \forall l \in L$

where $\underline{v}_{m_{l}}, \underline{z}_{\theta_{l}}$ are lower bounds and $\bar{v}_{m_{l}}, \bar{z}_{\theta_{l}}$ are upper bounds for their corresponding variables. McCormick envelopes for $v_{m_{l}}=v_{s_{l}} v_{r_{l}}$ are:

$v_{m_{l}} \geqslant \underline{v}_{s_{l}} v_{r}+\underline{v}_{r_{l}} v_{s_{l}}-\underline{v}_{s_{l}} \underline{v}_{r_{l}} \quad \forall l \in L$

$v_{m_{l}} \geqslant \bar{v}_{s_{l}} v_{r}+\bar{v}_{r_{l}} v_{s_{l}}-\bar{v}_{s_{l}} \bar{v}_{r_{l}} \quad \forall l \in L$

$v_{m_{l}} \leqslant \underline{v}_{s_{l}} v_{r}+\bar{v}_{r_{l}} v_{s_{l}}-\underline{v}_{s_{l}} \bar{v}_{r_{l}} \quad \forall l \in L$

$v_{m_{l}} \leqslant \bar{v}_{s_{l}} v_{r}+\underline{v}_{r_{l}} v_{s_{l}}-\bar{v}_{s_{l}} \underline{v}_{r_{l}} \quad \forall l \in L$

where $\underline{v}_{s_{l}}$ and $\underline{v}_{r_{l}}$ are lower bounds and $\bar{v}_{s_{l}}$ and $\bar{v}_{r_{l}}$ are upper bounds for their corresponding variables. McCormick envelopes for $z_{\theta_{l}}$ are:

$$
\begin{aligned}
& z_{\theta_{l}} \geqslant \cos \left(\frac{\overline{\theta_{l}}}{2}\right)\left(\theta_{l}+\overline{\overline{\theta_{l}}} \frac{\overline{2}}{2}\right)-\sin \left(\frac{\overline{\theta_{l}}}{2}\right) \quad \forall l \in L \\
& z_{\theta_{l}} \leqslant \cos \left(\overline{\frac{\theta_{l}}{2}}\right)\left(\theta_{l}-\frac{\overline{\theta_{l}}}{2}\right)+\sin \left(\frac{\overline{\theta_{l}}}{2}\right) \quad \forall l \in L
\end{aligned}
$$

where $\overline{\theta_{l}}$ is the upper bound of $\theta_{l}$. Constraints (64)-(73) are linear. Constraints (62) and (73) are valid for $0<\overline{\theta_{l}}<\pi / 2$. Bounds of the variables can be determined a priori. In Model E of SOC-ACOPF, we replace constraint (7) of optimization problem (1)-(10) by constraints (63)-(73). Accordingly, assumptions 1 and 2 are not required in Model E. Model E is summarized as following:

$$
\left\{\begin{array}{l}
\min _{\Omega} f\left(p_{n}, q_{n}, p_{o_{l}}, q_{o_{l}}\right) \\
\text { s.t. }(2)-(6),(8)-(10),(63)-(73)
\end{array}\right.
$$

Model E is convex because constraints (63)-(73) are linear. For the convexity of other constraints, please refer to the explanations of Model P in Section 2.1.

\section{Feasibility}

The proposed SOC-ACOPF models give relaxed solutions of the ACOPF problem. In case the AC feasibility is violated by the solutions of the derived SOC-ACOPF models, we propose here a heuristic algorithm to recover feasible solutions from the relaxed solutions. The heuristic technique is summarized in Algorithm 1. We use the relaxed solutions of the active power generation $p_{n}^{*}$ from the SOC-ACOPF models. If $p_{n}^{*}$ are feasible for all the ACOPF constraints, we can confirm that we have found the global optimal solution of ACOPF. Otherwise, we propose to fix $p_{n}=p_{n}^{*}$ for the cheap cost generators and take the $p_{n^{\prime}}$ of the marginal generator (the most expensive generator in $\left.N^{*}\right)$ as a variable. Where $N^{*} \subseteq N$ is a dynamic set initiated as $\forall n \in N^{*}, p_{n}^{*}>0$. $N^{*}$ is updated by removing the element $n^{\prime}$ which is the index of the dispatched generator with the highest marginal cost in each iteration. This process is repeated until the ACOPF is feasible in Algorithm 1. $c_{n}$ is the marginal cost of generator at node $n . i_{\max }$ is the maximum number of allowed iterations. We show in Section 4 of this paper that the feasible solution can be recovered by this algorithm normally in few iterations.

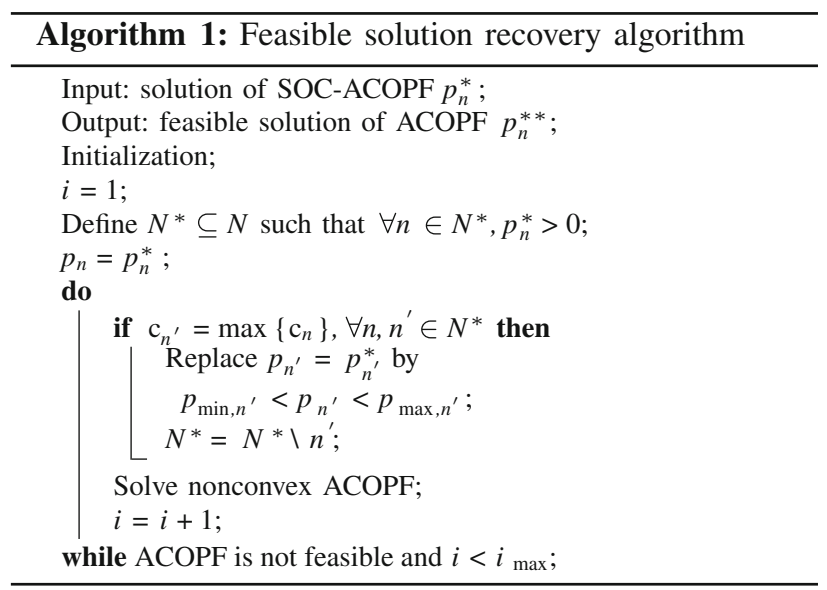




\section{Numerical results}

All the proposed SOC-ACOPF models (Model P, Model R, Model T and Model E) are implemented in GAMS and solved by MOSEK. A computer with $2.4 \mathrm{GHz}$ CPU and 8 GB RAM is deployed for the computations (except the computations in Section 4.2). It is worth to mention that voltage phase angle solutions can be obtained from solving the proposed SOC-ACOPF models directly since voltage phase angle is one of the decision variables in all the SOCACOPF models (see constraint (7) in Model P, constraints (17), (18) in Model R, constraints (40)-(45) in Model T and constraints (72), (73) in Model E). The convergence of MOSEK solver is guaranteed by the convexity of all the proposed SOC-ACOPF models. Solutions of ACOPF from MATPOWER [34] and LINDOGLOBAL are set as the benchmarks. The LINDOGLOBAL solver employs branch-and-cut methods to find the global optimal solution. MATPOWER uses MATLAB built-in interior point solver (MIPS) to solve nonconvex ACOPF. If a solution is not found, we denote the corresponding result as 'NA' (the LINDOGLOBAL solver in GAMS currently cannot solve optimization models with over 3000 variables and 2000 constraints). To compare with other SOC-ACOPF models in the literature, we also implement the model in reference [13] (denoted as Model 1 in the tables) and the model in reference [28] (denoted as Model 2 in the tables). The data for test case of 1354pegase and 2869pegase are from reference [35].

Since all the proposed SOC-ACOPF models are convex, solutions from MOSEK solver in GAMS for the models are global optimal. Please note the solutions of the proposed SOC-ACOPF models are global optimal for the corresponding SOC-ACOPF models. We do not mean that the optimal solutions from SOC-ACOPF models are global optimal for the nonconvex ACOPF model. Only the solutions from LINDOGLOBAL here can be regarded as the global optimal solution for the nonconvex ACOPF model. The solutions from MATPOWER are local optimal and are not guaranteed to be global optimal for the nonconvex ACOPF model. The slight differences of the solutions from the proposed SOC-ACOPF models are because the formulations are different and they have different feasible regions. In other words, the proposed SOC-ACOPF models are not equivalent with each other. This is why we use LINDOGLOBAL as a benchmark for the comparisons. The relative gap termination tolerance of the solution algorithm using by MOSEK to solve SOC-ACOPF models is $10^{-7}$. If the GAMS solution report of the MOSEK solver claims normal_completion, which means optimal solution is found, we will report the numerical results. The convexity of the proposed SOC-ACOPF models are further validated numerically here since the MOSEK solver in GAMS can only solve convex optimizal problems.

\subsection{Performance of SOC-ACOPF models}

\subsubsection{Base case}

The SOC-ACOPF objective values are listed in Table 1. The best results compared with LIDOGLOBAL are in bold. When LINDOGLOBAL cannot converge, we use the recovered best feasible solutions from Section 4.2 as the benchmark. The LINDOGLOBAL solver is able to find global solutions for IEEE 14-bus, IEEE 57-bus and IEEE 118-bus cases. For larger power networks, the number of variables and constraints exceed the limits of LINDOGLOBAL. The results of MATPOWER and LINDOGLOBAL are very close. All proposed SOC-ACOPF models give very close results as compared to MATPOWER and LINDOGLOBAL solutions. Normally, since our SOC-ACOPF models are relaxed models, the objective solutions are slightly lower than the solutions from LINDOGLOBAL which solves the nonconvex ACOPF model. Compared to LINDOGLOBAL results, the objective values of IEEE 14-bus case from Model T and Model E are bit higher. The reason is that the voltage phase angle constraint $0<\overline{\theta_{l}}<\pi / 2$ is included in Model $\mathrm{E}$ while this is not

Table 1 Objective value

\begin{tabular}{lrrrrrrrr}
\hline Case & \multicolumn{1}{l}{ Objective value $(\$)$} \\
\cline { 2 - 8 } & \multicolumn{1}{c}{ Model P } & \multicolumn{1}{c}{ Model R } & \multicolumn{1}{c}{ Model T } & \multicolumn{1}{c}{ Model E } & Model 1 & Model 2 & MATPOWER & LINDOGLOBAL \\
\hline IEEE 14 & $\mathbf{8 0 7 8 . 8 4}$ & 8075.22 & 8106.73 & 8092.32 & 8072.42 & 8073.16 & 8081.53 & 8081.54 \\
IEEE 57 & 41696.94 & $\mathbf{4 1 7 1 1 . 7 8}$ & $\mathbf{4 1 7 1 3 . 2 5}$ & $\mathbf{4 1 7 1 1 . 7 8}$ & 41673.10 & NA & 41737.79 & 41737.93 \\
IEEE 118 & 12919.50 & 129339.60 & $\mathbf{1 2 9 6 2 5 . 5 0}$ & 129376.00 & 129330.74 & 129325.68 & 129660.70 & 129660.54 \\
IEEE 300 & 719381.80 & 718301.60 & 721368.40 & 718546.27 & 718091.78 & $\mathbf{7 1 9 4 5 1 . 2 3}$ & 719725.11 & NA \\
1354pegase & $\mathbf{7 4 0 5 3 . 9 0}$ & 74096.14 & 74100.85 & 74040.99 & 74006.84 & 73974.56 & 74069.35 & NA \\
2869pegase & 133877.00 & 133875.40 & 133931.40 & $\mathbf{1 3 3 9 3 4 . 7 0}$ & 133866.95 & 133823.28 & 133999.29 & NA \\
\hline
\end{tabular}


Table 2 Computation CPU time

\begin{tabular}{|c|c|c|c|c|c|c|c|c|}
\hline \multirow[t]{2}{*}{ Case } & \multicolumn{8}{|c|}{ CPU time (s) } \\
\hline & Model P & Model R & Model T & Model E & Model 1 & Model 2 & MATPOWER & LINDOGLOBAL \\
\hline IEEE 14 & 0.08 & 0.06 & 0.12 & 0.09 & 0.08 & 0.07 & 0.11 & 0.20 \\
\hline IEEE 57 & 0.09 & 0.11 & 0.25 & 0.17 & 0.11 & NA & 0.12 & 2.31 \\
\hline IEEE 118 & 0.09 & 0.27 & 0.54 & 0.36 & 0.13 & 0.09 & 0.30 & 27.10 \\
\hline IEEE 300 & 0.25 & 1.29 & 1.48 & 0.64 & 0.25 & 0.22 & 0.48 & NA \\
\hline 1354pegase & 0.76 & 2.78 & 3.92 & 2.50 & 0.64 & 2.56 & 8.58 & NA \\
\hline 2869pegase & 1.97 & 10.94 & 11.47 & 9.72 & 1.23 & 6.82 & 18.66 & NA \\
\hline
\end{tabular}

necessary for the nonconvex ACOPF model in GAMS solved by LINDOGLOBAL (this can be also due to the accuracy tolerance differences of different optimization solvers).

The computation time results are listed in Table 2. Model $\mathrm{P}$ requires least computation time while Model $\mathrm{T}$ requires the most. This is because model complexity increases as we increase model accuracy. The accuracy improvement is validated by the solution results in Table 1. All proposed SOC-ACOPF models are computationally competitive with MATPOWER. The proposed SOCACOPF models require much less computation time as compared to MATPOWER for large-scale network cases (see the results of 1354pegase and 2869pegase cases in Table 2). Our proposed SOC-ACOPF models are valid for both radial and mesh power networks.

To compare the computational performance of different SOCP-based ACOPF formulations, we have also implemented the ACOPF models in [13] and [28]. The results are listed in Tables 1 and 2. It is worth to mention that the model in reference [28] takes much longer time than our proposed SOC-ACOPF models for GAMS to generate the executable model format to the solver though the solver CPU time is short. In general, the model in [13] has the least number of constraints and requires less computational time. However, this model is not valid for mesh networks because there is no voltage phase angle constraint in this model (the results from the model in [13] are relaxed solutions for mesh networks). For the model in [28], MOSEK in GAMS cannot converge for the IEEE 57-bus test case. These results show stronger robust performance of our SOC-ACOPF models compared with the other two convex ACOPF models in the literature.

\subsubsection{Power load scenarios}

We evaluate the performance of the proposed SOCACOPF models under different power load scenarios and compare the results with MATPOWER results. The incremental power load scenarios are generated from $10 \%$ to $100 \%$ of the power loads (both active power and reactive power) in the base case. The SOC-ACOPF and ACOPF models are solved repeatedly by using different power load parameters. To demonstrate the relaxation performance of the proposed SOC-ACOPF models, we also calculate and plot the maximum value of relaxation gaps $G_{o_{l}}$. The maximum relaxation gap $G_{o_{l}}$ is calculated by:

$$
G_{o_{l}}=\max _{l}\left\{p_{o_{l}}-\frac{p_{s_{l}}^{2}+q_{s_{l}}^{2}}{V_{s_{l}}} R_{l}\right\}
$$

Small values of $G_{o_{l}}$ mean better AC feasibility. The results are shown in Figs. 1, 2, 3, 4, 5, 6, 7, 8, 9, 10, 11 and 12. For IEEE 14-bus, IEEE 57-bus, IEEE 118-bus and IEEE 300 -bus cases, $G_{O_{l}}<10^{-6}$ is valid for all the power load scenarios. For power load scenarios when MATPOWER cannot converge, the MOSEK solver is convergent for our proposed models however with large relaxation gaps (see in Fig. 10 the results of relaxation gap for power load ratio $10 \%$ ). These results show strong robustness of the proposed SOC-ACOPF models over the power load scenarios.

\subsubsection{Congested power networks}

The network congestions are caused by reducing the capacity of transmission lines to be $80 \%$ of the power flow solutions in the base case. Each line is congested sequentially. We summarize the statistics of the results in Table 3.

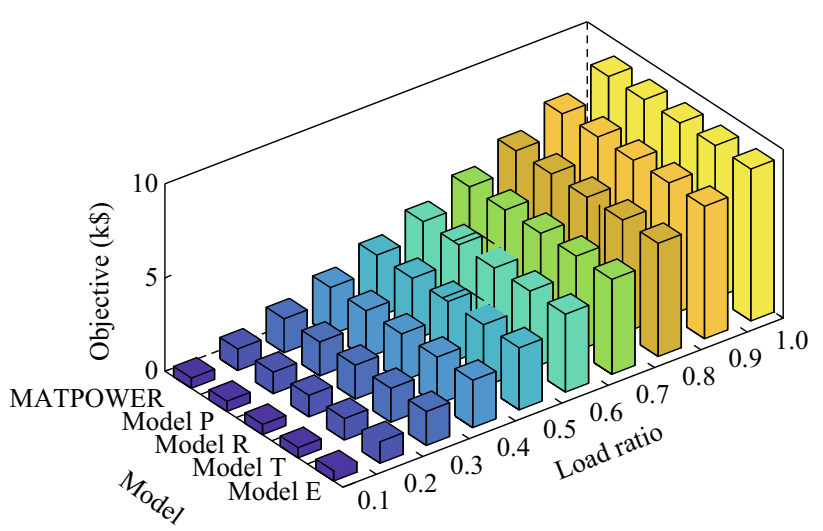

Fig. 1 Objectives of IEEE 14-bus for different power load scenarios 


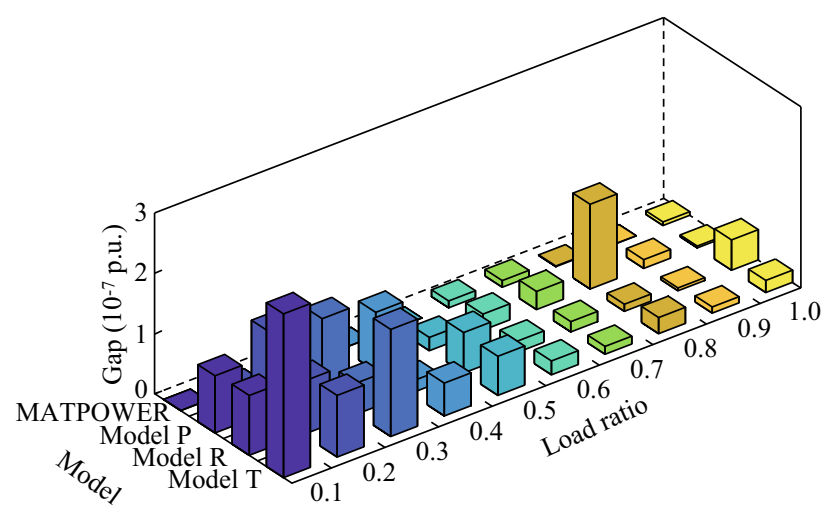

Fig. 2 Relaxation gaps of IEEE 14-bus for different power load scenarios

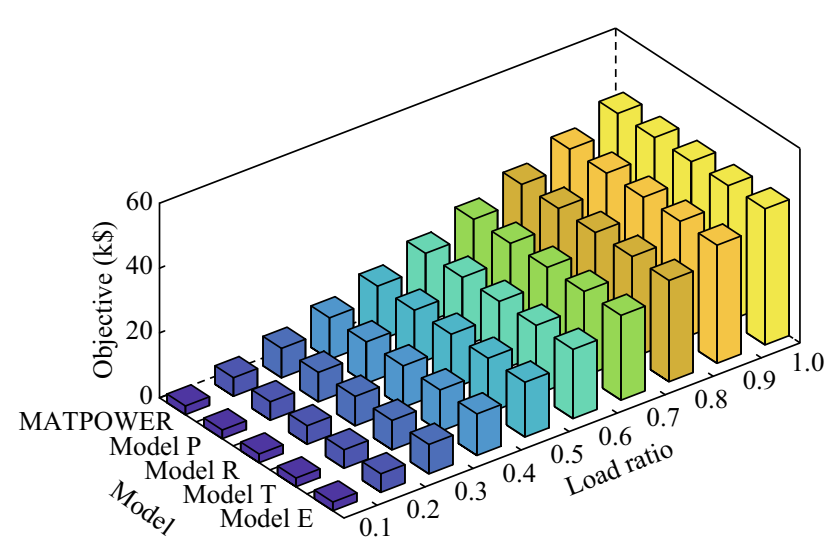

Fig. 3 Objectives of IEEE 57-bus for different power load scenarios

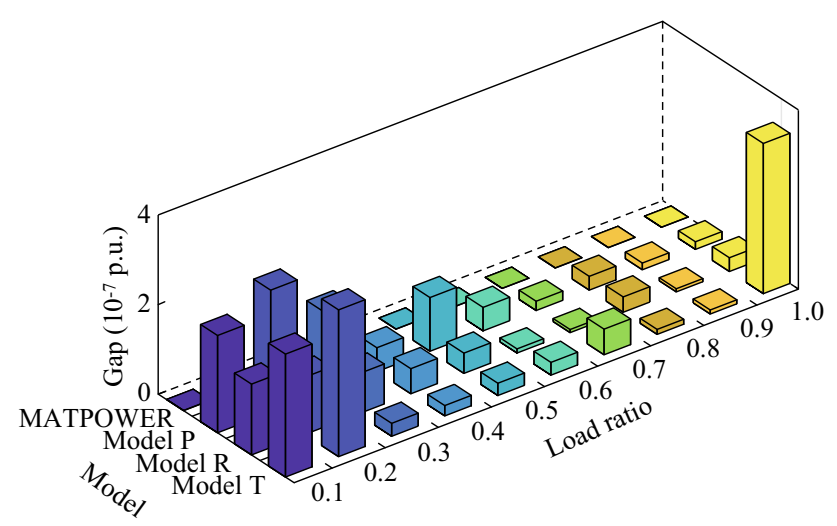

Fig. 4 Relaxation gaps of IEEE 57-bus for different power load scenarios

When some critical lines are congested, both MATPOWER and GAMS cannot converge. All the proposed SOCACOPF models are more robust than the nonconvex ACOPF model in MATPOWER. The convergence performance of Model $\mathrm{P}$ is very close to the nonconvex ACOPF model in MATPOWER. These results show that

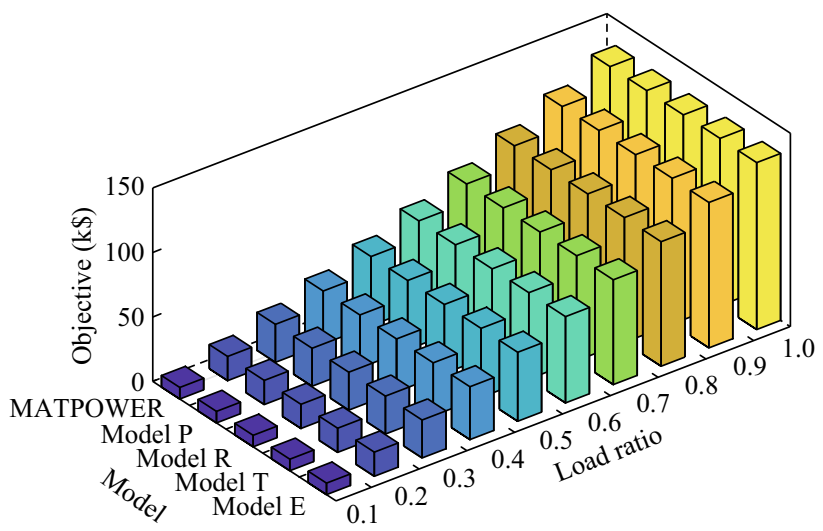

Fig. 5 Objectives of IEEE 118-bus for different power load scenarios

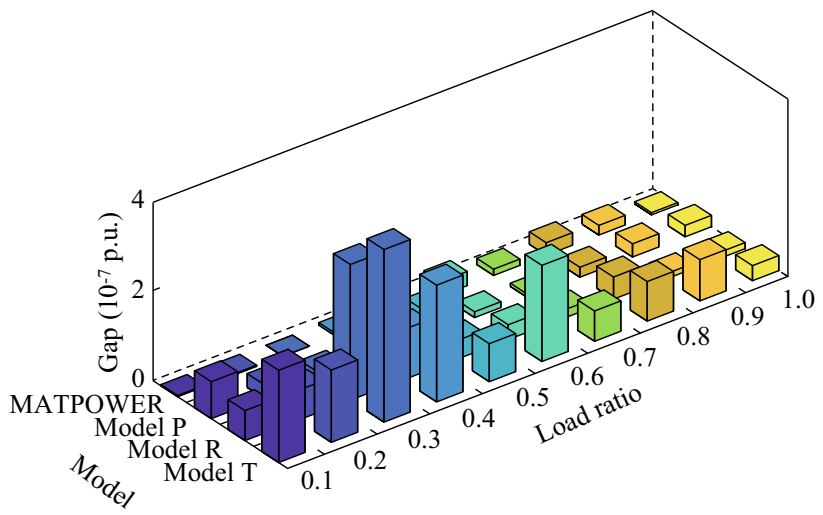

Fig. 6 Relaxation gaps of IEEE 118-bus for different power load scenarios

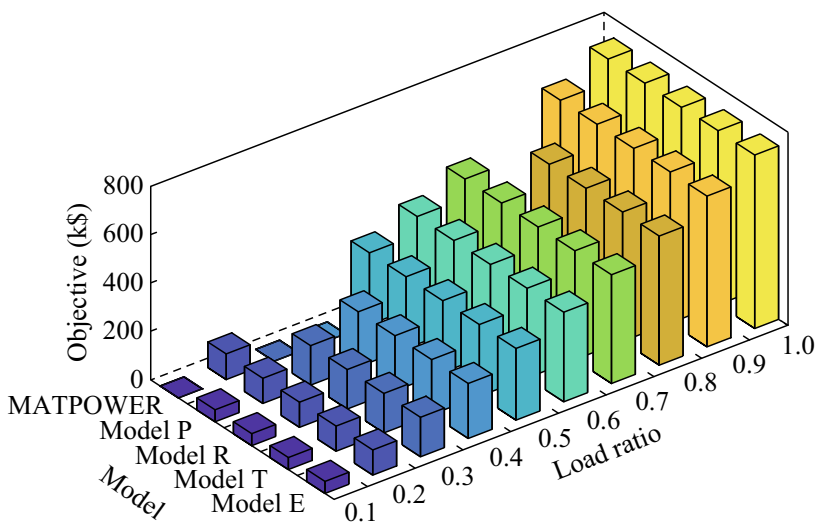

Fig. 7 Objectives of IEEE 300-bus for different power load scenarios

the improvements (robustness) of the proposed SOCACOPF models compared with Model P are prominent.

\subsection{Feasible solution}

The heuristic algorithm described in Section 3 for recovering a feasible solution is validated numerically in 


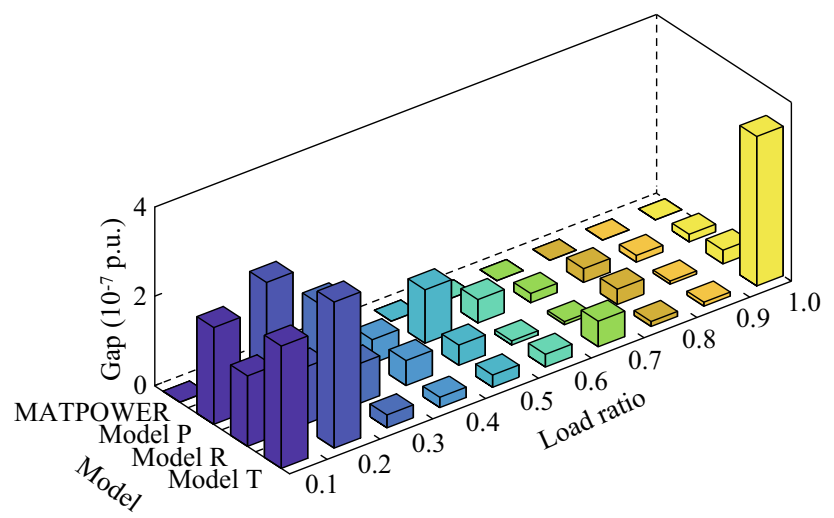

Fig. 8 Relaxation gaps of IEEE 300-bus for different power load scenarios

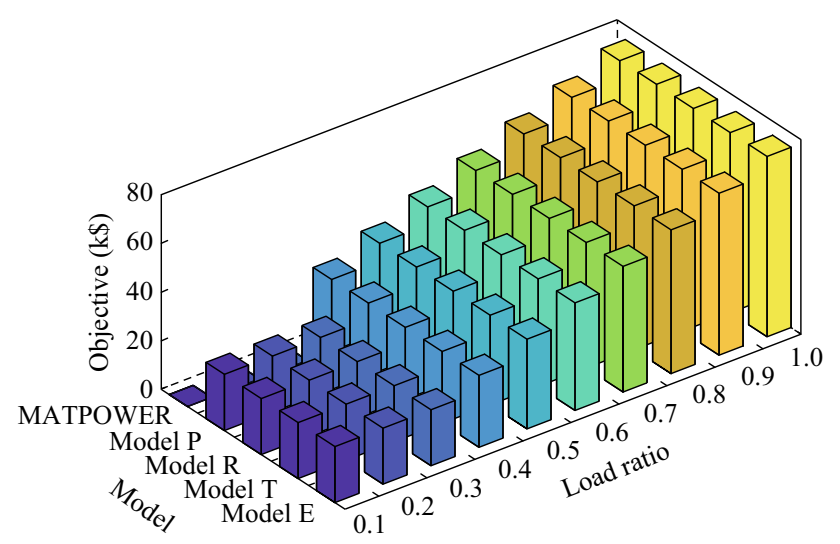

Fig. 9 Objectives of 1354pegase for different power load scenarios

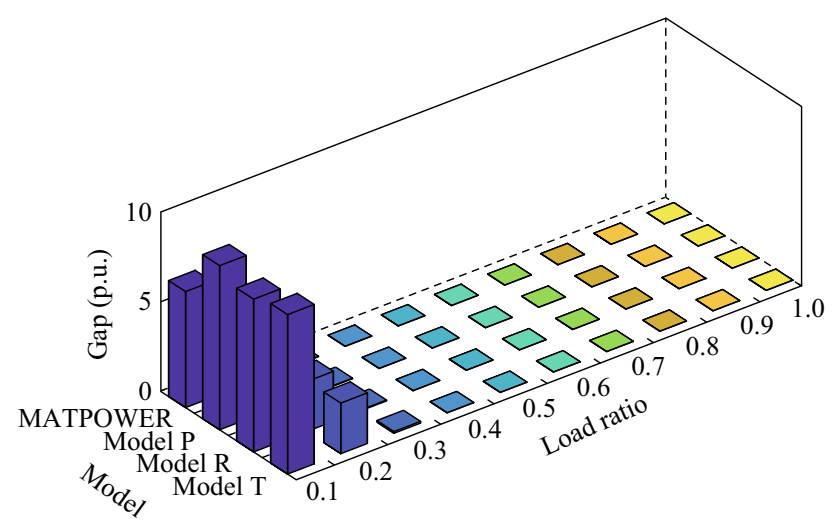

Fig. 10 Relaxation gaps of 1354pegase for different power load scenarios

this section. We use the IPOPT solver in GAMS to solve the nonconvex ACOPF model. A desktop with $3.4 \mathrm{GHz}$ CPU and 32 GB RAM is used to implement Algorithm 1. For all the relaxed solutions of SOC-ACOPF models listed in Table 1, the feasible solutions are recovered within two iterations. The objective function values of the recovered

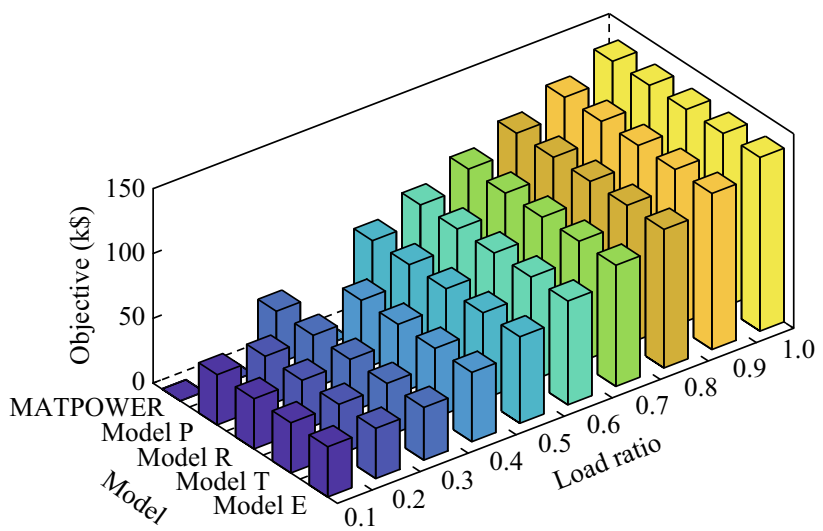

Fig. 11 Objectives of 2869pegase for different power load scenarios

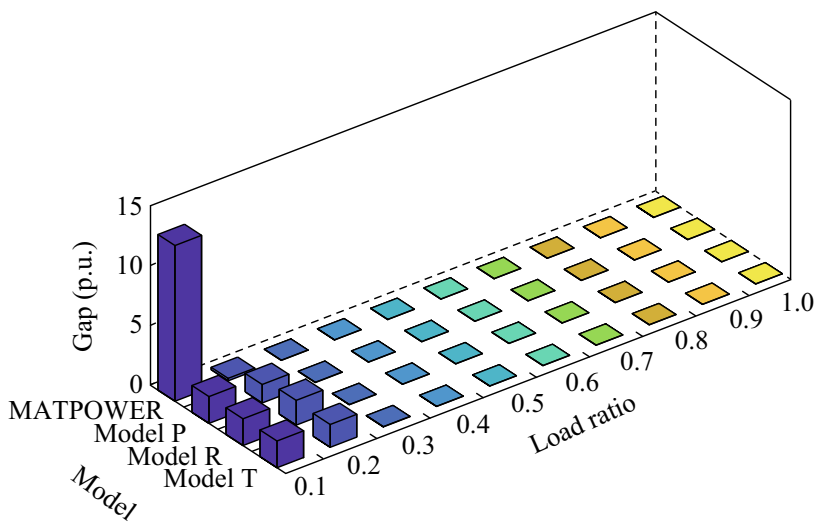

Fig. 12 Relaxation gaps of 2869pegase for different power load scenarios

feasible solutions are listed in Table 4. The CPU time required for the computation of the feasible solutions are reported in Table 5. The feasible solutions from the models in [13] and [28] are also recovered and reported. Feasible solutions recovered from our SOC-ACOPF models are better than [13] and [28]. For 1354pegase and 2869pegase test cases, feasible solutions with lower objective function values than the solutions from MATPOWER are recovered. The disadvantage of the proposed heuristic feasible solution recovery algorithm is that the computation time is larger than solving the nonconvex ACOPF model by MATPOWER. However, this algorithm is still useful when computation time is not strictly constrained considering some recovered feasible solutions are better than the solutions obtained from MATPOWER. It is also worth to mention that, since IPOPT is an open source solver, it may not be suitable to compare the CPU time of IPOPT with the MIPS solver in MATLAB which is commercial software. The major reason of larger CPU time is due to the iterative nature of the heuristic feasible solution recovery algorithm. 
Table 3 Convergence performance in congested power networks

\begin{tabular}{|c|c|c|c|c|c|c|}
\hline \multirow[t]{2}{*}{ Case } & \multicolumn{5}{|c|}{ Number of convergent solutions } & \multirow[t]{2}{*}{ Total number of transmission lines } \\
\hline & Model P & Model R & Model T & Model E & MATPOWER & \\
\hline IEEE 14 & 20 & 20 & 20 & 20 & 19 & 20 \\
\hline IEEE 57 & 79 & 80 & 77 & 80 & 80 & 80 \\
\hline IEEE 118 & 186 & 186 & 185 & 186 & 182 & 186 \\
\hline IEEE 300 & 357 & 372 & 350 & 359 & 344 & 411 \\
\hline 1354pegase & 1391 & 1723 & 1655 & 1665 & 1366 & 1991 \\
\hline 2869pegase & 3897 & 4278 & 4221 & 4238 & 3409 & 4582 \\
\hline
\end{tabular}

Table 4 Objective values of recovered feasible solution

\begin{tabular}{lrrrrrrrr}
\hline Case & \multicolumn{1}{l}{ Objective value $(\$)$} \\
\cline { 2 - 8 } & \multicolumn{1}{c}{ Model P } & \multicolumn{1}{c}{ Model R } & \multicolumn{1}{c}{ Model T } & \multicolumn{1}{c}{ Model E } & Model 1 & Model 2 & MATPOWER & LINDOGLOBAL \\
\hline IEEE 14 & $\mathbf{8 0 8 1 . 6 1}$ & $\mathbf{8 0 8 1 . 6 3}$ & 8106.73 & 8091.10 & $\mathbf{8 0 8 1 . 6 3}$ & 8081.63 & 8081.53 & 8081.54 \\
IEEE 57 & $\mathbf{4 1 7 3 8 . 1 1}$ & $\mathbf{4 1 7 3 8 . 1 5}$ & $\mathbf{4 1 7 3 8 . 1 4}$ & $\mathbf{4 1 7 3 8 . 1 5}$ & $\mathbf{4 1 7 3 8 . 1 5}$ & NA & 41737.79 & 41737.93 \\
IEEE 118 & $\mathbf{1 2 9 6 6 0 . 9 2}$ & 129668.43 & 129668.29 & 129667.12 & 129668.43 & 129670.80 & 129660.70 & 129660.54 \\
IEEE 300 & 719900.86 & 719536.08 & $\mathbf{7 1 9 5 1 6 . 7 9}$ & 719526.16 & 719522.19 & 720335.29 & 719725.11 & NA \\
1354pegase & $\mathbf{7 4 0 6 4 . 7 7}$ & $\mathbf{7 4 0 6 4 . 7 7}$ & $\mathbf{7 4 0 6 4 . 7 7}$ & $\mathbf{7 4 0 6 4 . 7 7}$ & $\mathbf{7 4 0 6 4 . 7 7}$ & $\mathbf{7 4 0 6 4 . 7 7}$ & 74069.35 & NA \\
2869pegase & $\mathbf{1 3 3 9 8 7 . 7 6}$ & 133991.67 & 133991.67 & 133991.67 & 133991.67 & 133991.67 & 133999.29 & NA \\
\hline
\end{tabular}

Table 5 Computation time of feasible solution recovery algorithm

\begin{tabular}{lrrrrrrrr}
\hline Case & \multicolumn{1}{l}{ CPU time $(\mathrm{s})$} & & & & \\
\cline { 2 - 8 } & Model P & Model R & Model T & Model E & Model 1 & Model 2 & MATPOWER & LINDOGLOBAL \\
\hline IEEE 14 & 0.11 & 0.18 & 0.26 & 0.12 & 0.22 & 0.25 & 0.11 & 0.20 \\
IEEE 57 & 1.05 & 1.50 & 1.22 & 1.17 & 1.59 & NA & 0.12 & 0.30 \\
IEEE 118 & 1.44 & 2.41 & 1.37 & 3.03 & 4.08 & 3.47 & 14.53 & NA \\
IEEE 300 & 14.55 & 24.56 & 15.13 & 131.55 & 15.28 & 13.42 & NA \\
1354pegase & 45.90 & 41.77 & 69.55 & 33.91 & 58.61 & 19.45 & 18.66 & NA \\
2869pegase & 234.61 & 769.156 & 140.58 & 525.00 & 203.69 & 127.30 & \\
\hline
\end{tabular}

\section{Conclusion}

Three second-order cone models (Model R, Model T and Model E) for ACOPF are proposed using convex relaxation and approximation techniques. Compared with other SOCP-based ACOPF formulations in the literature, our formulations are valid for both mesh and radial power networks. The numerical results show that the proposed SOC-ACOPF models can give accurate results. Though the bilinear transformation based derivations lead to more constraints in the proposed SOC-ACOPF models compared with Model P, the accuracy improvement is achieved with similar computation time as compared to MATPOWER. The quality of results with respect to the global optimal solution is also checked using LINDOGLOBAL solver in GAMS. The computation results for various power load scenarios show robust performance of the proposed SOCACOPF models. An interesting observation from the results of test cases with low power load levels when MATPOWER cannot converge is that high relaxation gap values from the solutions of the proposed SOC-ACOPF models can actually be regarded as indicators of non-convergence of the original nonconvex ACOPF model. In these cases, the objective value solutions from the proposed SOC-ACOPF models can serve as lower bounds of the original nonconvex ACOPF model.

A computational comparison of different SOCP-based ACOPF formulations shows the strong convergence 
performance of the proposed SOC-ACOPF models. To recover feasible solutions from the relaxed solutions of the proposed SOC-ACOPF models, we develop a heuristic feasible solution recovery algorithm. This algorithm is capable of recovering the feasible solutions from all the relaxed solutions of the proposed SOC-ACOPF models in the test cases. Another key observation from our research is that, instead of seeking one single model to satisfy all test cases under all scenarios, it is perhaps more realistic to provide multiple models or methods, which can be valuable or applicable for the power network operators to deal with many different test cases or operational situations. This is reasonable considering the large-scale changing nature of power network parameters (such as power loads and network congestions) in the real-world operations. Considering uncertainties for the proposed SOC-ACOPF models can be good future work.

Acknowledgements The authors would like to express special thanks to the editors' and reviewers' great efforts of reviewing and improving this paper.

Open Access This article is distributed under the terms of the Creative Commons Attribution 4.0 International License (http:// creativecommons.org/licenses/by/4.0/), which permits unrestricted use, distribution, and reproduction in any medium, provided you give appropriate credit to the original author(s) and the source, provide a link to the Creative Commons license, and indicate if changes were made.

\section{References}

[1] Momoh JA, Adapa R, El-Hawary ME (1999) A review of selected optimal power flow literature to 1993 Part I: nonlinear and quadratic programming approaches. IEEE Trans Power Syst 14(1):96-104

[2] Momoh JA, El-Hawary M, Adapa R (1999) A review of selected optimal power flow literature to 1993 Part II: Newton, linear programming and interior point methods. IEEE Trans Power Syst 14(1):105-111

[3] Sun G, Chen S, Wei Z (2017) Multi-period integrated natural gas and electric power system probabilistic optimal power flow incorporating power-to-gas units. J Mod Power Syst Clean Energy 5(3):412-423

[4] Cao J, Yan Z, Xu X (2016) Optimal power flow calculation in AC/DC hybrid power system based on adaptive simplified human learning optimization algorithm. J Mod Power Syst Clean Energy 4(4):690-701

[5] Niu M, Wan C, Xu Z (2014) A review on applications of heuristic optimization algorithms for optimal power flow in modern power systems. J Mod Power Syst Clean Energy 2(4):289-297

[6] Cai H, Chung C, Wong K (2008) Application of differential evolution algorithm for transient stability constrained optimal power flow. IEEE Trans Power Syst 23(2):719-728

[7] Overbye TJ, Cheng X, Sun Y (2004) A comparison of the AC and DC power flow models for LMP calculations. In:
Proceedings of the 37th annual Hawaii international conference on system sciences, Big Island, USA, 5-8 January 2004, 9 pp

[8] Amin SM, Wollenberg BF (2005) Toward a smart grid: power delivery for the 21st century. IEEE Power Energy Mag 3(5):34-41

[9] Abdelouadoud S, Girard R, Neirac FP (2015) Optimal power flow of a distribution system based on increasingly tight cutting planes added to a second order cone relaxation. Int $\mathrm{J}$ Electr Power Energy Syst 69:9-17

[10] Lipka P, Oren S, O’Neill R (2016) Running a more complete market with the SLP-IV-ACOPF. IEEE Trans Power Syst 32(2):1139-1148

[11] Liu Y, Li J, Wu L (2017) Distribution system restructuring: distribution LMP via unbalanced ACOPF. IEEE Trans Smart Grid 9(5):4038-4048

[12] MOSEK (2018) The MOSEK optimization software. http:// www.mosek.com. Accessed 1 March 2018

[13] Farivar M, Low SH (2013) Branch flow model: relaxations and convexification: Part I. IEEE Trans Power Syst 28(3):2554-2564

[14] Gan L, Li N, Topcu U (2015) Exact convex relaxation of optimal power flow in radial networks. IEEE Trans Autom Control 60(1):72-87

[15] Baradar M, Hesamzadeh MR (2015) AC power flow representation in conic format. IEEE Trans Power Syst 30(1):546-547

[16] Jabr RA (2006) Radial distribution load flow using conic programming. IEEE Trans Power Syst 21(3):1458-1459

[17] Yuan Z, Hesamzadeh MR, Biggar D (2016) Distribution locational marginal pricing by convexified ACOPF and hierarchical dispatch. IEEE Trans Smart Grid 9(4):3133-3142

[18] Yuan Z, Hesamzadeh MR (2017) Hierarchical coordination of TSO-DSO economic dispatch considering large-scale integration of distributed energy resources. Appl Energy 195:600-615

[19] Yuan Z, Hesamzadeh MR (2017) A modified benders decomposition algorithm to solve second-order cone AC optimal power flow. IEEE Trans Smart Grid. https://doi.org/10.1109/ TSG.2017.2776407

[20] Bai X, Wei H, Fujisawa K (2008) Semidefinite programming for optimal power flow problems. Int J Electr Power Energy Syst 30(6):383-392

[21] Lavaei J, Low SH (2012) Zero duality gap in optimal power flow problem. IEEE Trans Power Syst 27(1):92-107

[22] Lesieutre BC, Molzahn DK, Borden AR et al (2011) Examining the limits of the application of semidefinite programming to power flow problems. In: Proceedings of 49th annual Allerton conference on communication, control, and computing, Monticello, USA, 28-30 September 2011, pp 1492-1499

[23] Molzahn D, Holzer J, Lesieutre B (2013) Implementation of a large-scale optimal power flow solver based on semidefinite programming. IEEE Trans Power Syst 28(4):3987-3998

[24] Lavaei J, Tse D, Zhang B (2014) Geometry of power flows and optimization in distribution networks. IEEE Trans Power Syst 29(2):572-583

[25] Low S (2014) Convex relaxation of optimal power flow, Part I: formulations and equivalence. IEEE Trans Control Netw Syst 1(1):15-27

[26] Low SH (2014b) Convex relaxation of optimal power flow, Part II: exactness. IEEE Trans Control Netw Syst 1(2):177-189

[27] Kocuk B, Dey SS, Sun XA (2016) Inexactness of SDP relaxation and valid inequalities for optimal power flow. IEEE Trans Power Syst 31(1):642-651

[28] Coffrin C, Hijazi HL, Hentenryck PV (2016) The QC relaxation: a theoretical and computational study on optimal power flow. IEEE Trans Power Syst 31(4):3008-3018 
[29] O'Neill RP, Castillo A, Cain MB (2012) The IV formulation and linear approximations of the AC optimal power flow problem. FERC Staff Technical Paper

[30] O'Neill RP, Castillo A, Cain MB (2012) The computational testing of AC optimal power flow using the current voltage formulations. FERC Staff Technical Paper

[31] Kocuk B, Dey SS, Sun XA (2016) Strong SOCP relaxations for the optimal power flow problem. Oper Res 64(6):1177-1196

[32] Ghamkhari M, Sadeghi-Mobarakeh A, Mohsenian-Rad H (2016) Strategic bidding for producers in nodal electricity markets: a convex relaxation approach. IEEE Trans Power Syst 32(3):2324-2336

[33] McCormick GP (1976) Computability of global solutions to factorable nonconvex programs: Part I convex underestimating problems. Math Program 10(1):147-175

[34] Zimmerman RD, Murillo-Sánchez CE, Thomas RJ (2011) MATPOWER: steady-state operations, planning, and analysis tools for power systems research and education. IEEE Trans Power Syst 26(1):12-19

[35] Fliscounakis S, Panciatici P, Capitanescu F (2013) Contingency ranking with respect to overloads in very large power systems taking into account uncertainty, preventive, and corrective actions. IEEE Trans Power Syst 28(4):4909-4917

Zhao YUAN received B.E. degree in electrical engineering from Hebei University of Technology in 2011, and double M.E. degrees in electrical engineering from Huazhong University of Science and Technology and in renewable energy from ParisTech in 2014. He was awarded the national second prize in 2010 China Undergraduate Contest of Mathematical Modelling by China Society for Industrial and Applied Mathematics. He is currently pursuing the Ph.D. degree in the Department of Electric Power and Energy Systems of KTH Royal Institute of Technology, Sweden. His research work initiates and contributes to two technical patents in Sweden. His research interests include optimal power flow, power system operation and electricity market.

Mohammad Reza HESAMZADEH received his Docent degree from KTH Royal Institute of Technology, Sweden, and his Ph.D. degree from Swinburne University of Technology, Australia, in 2013 and 2010 respectively. He was a post-doctoral fellow at KTH in 2010-2011 where he is currently a faculty member. He is a member of International Association for Energy Economics (IAEE) and a Member of CIGRE, Sweden Section. His special fields of interests include electricity market modeling, analysis, and design and mathematical modelling and computing. 